\title{
The diagnostic accuracy by a general practitioner of video-otoscopy collected by a health care facilitator compared to traditional otoscopy
}

Thorbjörn Lundberg, M.D., Ph.D.

Corresponding author

Department of Public Health and Clinical Medicine, Family medicine, Umea University, S-901 87 Umea, Sweden. Tel: +4690-78500 00. Fax: +46 90-77 6883

thorbjorn.lundberg@umu.se

Leigh Biagio de Jager, Ph.D.

1. Department of Speech-Language Pathology and Audiology, University of Pretoria, Pretoria, South Africa

De Wet Swanepoel, Ph.D.

1. Department of Speech-Language Pathology and Audiology, University of Pretoria, Pretoria, South Africa

2. Ear Science Institute Australia, Subiaco, Australia

3. Ear Sciences Centre, School of Surgery, The University of Western Australia, Nedlands, Australia

Claude Laurent, M.D., Ph.D.

1. Department of Clinical Science, ENT-unit, Umea University, Umea, Sweden

2. Department of Speech-Language Pathology and Audiology, University of Pretoria, Pretoria, South Africa

\section{Abstract}

Objective: Video-otoscopy is rapidly developing as a new method to diagnose common ear disease and can be performed by trained health care facilitators as well as by clinicians. This study aimed to compare remote asynchronous assessments of video-otoscopy with otoscopy performed by a general practitioner.

Method: Children, aged 2-16 years, attending a health center in Johannesburg, South Africa, were examined. An otologist performed otomicroscopy and a general practitioner performed otoscopy. Video-otoscopy was performed by a health care facilitator and video sequences were stored on a server for assessment by the same general practitioner 4 and 8 weeks later. At all examinations, a diagnosis was set and the tympanic membrane appearance was graded using the OMgrade-scale. The otologist's otomicroscopic diagnosis was set as reference standard to compare the accuracy of the two otoscopic methods.

Results: Diagnostic agreement between otologist's otomicroscopic examination and the general practitioner's otoscopic examination was substantial (kappa 0.66). Agreement 
between onsite otomicroscopy and the general practitioners asynchronous video assessments were also substantial (kappa 0.70 and 0.80 ).

Conclusion: Video-otoscopy performed by a health care facilitator and assessed asynchronously by a general practitioner had similar or better accuracy compared to face-toface otoscopy performed by a general practitioner.

\section{Keywords}

Otoscopy; Video-otoscopy; Otitis media; General practice; Telemedicine

\section{Introduction}

Otitis media $(\mathrm{OM})$ is a common disease and may lead to lifelong sequelae $(1,2)$. Approximately 740 million people world-wide will be affected annually by acute otitis media (AOM) or chronic suppurative otitis media (CSOM) and by the age of 10 at least $90 \%$ of children will have experienced otitis media with effusion $(\mathrm{OME})(2,3)$.

Diagnosis of $\mathrm{OM}$ is based on a review of symptoms together with assessment of the tympanic membrane (TM) and middle ear status. However, diagnostic inaccuracy among pediatricians and general practitioners is a clinical challenge that may lead to over-diagnosis $(4,5)$. At the same time, access to health care differs around the world, with some areas lacking health personnel for accurate diagnosis (6). Pediatricians and general practitioners usually use handheld otoscopes for diagnosis of middle ear disease, whereas otologists prefer otomicroscopes. However, rapid technological development has led to the introduction of cost-effective video-otoscopes to facilitate and improve the diagnostic outcome in otoscopy. Video-otoscopes allow capturing multiple images or video on computer or smartphone on the condition of the ear canal and TM with the possibility of interpretation either onsite or 
remotely (7-9). Captured video-sequences allow for repeated back and forward examination of the recording (7).

\subsection{Aim}

To evaluate the diagnostic accuracy of remote assessment of video-otoscopy recordings captured by a health care facilitator and diagnosed by a general practitioner as compared with traditional otoscopy by a general practitioner.

\section{Method}

The Institutional Ethics Committee at the University of Pretoria, Pretoria, South Africa approved of the study.

Caregivers of children aged two to 16 years attending a primary health care clinic outside Johannesburg (Witkoppen Health and Welfare clinic), were offered a free ear examination. Over a two-week period, 140 children were recruited $(7,10,11)$ after informed consent.

\subsection{Reference standard}

An experienced otologist ( $>35$ years of practice) first examined the children's ears using a Leica M525 F40 surgical otomicroscope with a 6:1 zoom magnification (1.2 to 12.8x) and a 300-watt xenon fiber optic illumination. Cerumen was removed manually if needed, and when cerumen could not be removed sufficiently to visualize the TM, the diagnoses were considered as not possible to determine (NPD). The otologist examined both ears and gave one diagnosis for each ear (reference standard). 


\subsection{Index tests}

The child was then examined by a general practitioner (GP) (>15 years of practice) who performed an otoscopic examination of both ears using a hand-held, Heine K100 Diagnostic otoscope, without knowledge of the otologists' diagnoses.

Video-otoscopy was finally performed by a trained health care facilitator who completed recordings of video-clips, $>30$ seconds in length that were stored on a laptop. The health care facilitator had a two-day training period on conducting video-otoscopy, provided by the otologist (7). The video-otoscopic recordings were made with a Dino-Lite Pro Earscope with a LED light, a magnification of 10-20x, a frame rate of 30 frames/sec and a 1.3-megapixel resolution. The Dino-Lite video-otoscope was attached via a USB cable to a Lenovo ThinkPad 2.0 running Windows 7 via 2.0 interface or a Macbook pro running OSX v10.7.5. DinoCapture 2.0 software (AnMo Electronics Corporation) version 1.2.7 was used to record and view the video-otoscopic recordings. The recordings were saved onto a laptop as MOVor WMV-files (Macbook Pro and PC) and ranged from 0.85 to $7.61 \mathrm{MB}$ size (mean $=3.6$ MB).

All examinations, including micro-otoscopy, otoscopy and video-otoscopy, were performed consecutively the day the child attended the health center.

Video-otoscopic recordings were uploaded to a secure virtual database (Dropbox). The GP then accessed the server from Umeå University, Sweden, on two occasions 4 weeks and 8 weeks after onsite assessment (video-otoscopy 1 and 2), using a 24 inch Apple LED Cinema Display connected to a Macbook Pro. Recordings were anonymous and the order randomized. The GPs' assessments were recorded on an Excel spreadsheet and uploaded to the Dropbox server, which was managed by an independent investigator. 


\subsection{Diagnosis}

The diagnoses, made both synchronously and asynchronously were: Normal tympanic membrane (n-TM), otitis media with effusion (OME), acute otitis media (AOM) and chronic suppurative otitis media (CSOM) (Table 1) (12). If the status of TM's could not be determined at otoscopy or at any of the two asynchronous video-otoscopic assessments (due to lack of co-operation, obstructing cerumen or poor image quality) it was labeled as NPD.

Table 1

Diagnosis of otitis media

\begin{tabular}{ll}
\hline Diagnosis & \multicolumn{1}{c}{ Description } \\
\hline Normal & A translucent TM in a fairly normal position without signs of middle ear fluid \\
OME & $\begin{array}{l}\text { A translucent TM with visible middle ear fluid or an opaque TM in a fairly normal position } \\
\text { and no clinical signs of acute infection }\end{array}$ \\
AOM $\quad \begin{array}{l}\text { An opaque and bulging TM together with clinical signs of acute infection such as otalgia } \\
\text { and fever }\end{array}$ & A TM with chronic perforation \\
NPD & Ear status not possible to determine
\end{tabular}

We also used a validated image based grading scale, OMgrade (13), for grading of the TM appearance (Table 2). This grading scale is based on 6 grades with a subdivision of grade 5 . The scale is based on assessing transparency and position of the TM. 


\section{Table 2}

The revised OMgrade scale and a description of each scale step (13).

\begin{tabular}{|c|c|c|}
\hline Grade & Sub-grade & Description \\
\hline 0 & 0 & Transparent TM in a normal position \\
\hline 1 & 1 & $\begin{array}{l}\text { Transparent TM in a normal position or slightly retracted with a fluid } \\
\text { level or fluid filled middle ear }\end{array}$ \\
\hline 2 & 2 & $\begin{array}{l}\text { Transparent TM in a normal position or slightly retracted with a } \\
\text { turbid/opaque fluid level }\end{array}$ \\
\hline 3 & 3 & Opaque appearance of the TM in a fairly normal position \\
\hline 4 & 4 & Opaque appearance of the TM and bulging \\
\hline \multirow[b]{2}{*}{5} & $5 B$ & Opaque appearance of the TM with bullous formations \\
\hline & $5 C$ & $\begin{array}{l}\text { Contourless TM with a wet appearance and swollen keratin patches, } \\
\text { w/wo pulsating pus from small perforation }\end{array}$ \\
\hline 6 & 6 & $\begin{array}{l}\text { TM perforation, retraction pocket or cholesteatoma, w/wo purulent } \\
\text { discharge, previous ear surgery and TM grommets }\end{array}$ \\
\hline
\end{tabular}

w/wo: with or without

\subsection{Exclusions}

All cases with missing data or ears not possible to determine (NPD) at each diagnostic step were excluded. Not possible to diagnose (NPD) was due to ears with obstructing wax, a child not co-operating, or a video sequence with low quality.

\subsection{Data analysis}

The diagnostic concordance for otoscopy and video-otoscopy was measured with percentage of agreement and Cohen's kappa using the otomicroscopy as reference standard. We used Landis and Koch characterizations of the kappa values: $<0=$ no agreement, $0-0.20=$ slight, $0.21-0.40=$ fair, $0.41-0.60=$ moderate, $0.61-0.80=$ substantial, $0.81-1=$ almost perfect agreement.

We used SPSS v24 for calculations of percentage of agreement, Cohen's kappa, frequencies and cross-tabulations. For the OMgrade calculations with a high number of ordinal variables the weighted kappa was calculated using StatsToDo online calculator:

http://www.statstodo.com/CohenKappa_Pgm.php. 
Sensitivity, specificity and Chi2-test were calculated using MedCalc online calculator:

https://www.medcalc.org/calc/diagnostic_test.php.

\section{Results}

One hundred and forty children were recruited during a two-week period and 280 ears were examined. Seventy-four percent of all examined ears (280 ears) were diagnosed as normal TM's, $10 \%$ as OME, $0.7 \%$ AOM, $13 \%$ as CSOM and $10.4 \%$ as missing or NPD. The distribution of diagnoses in included ears can be seen in Table 3. The 105 ears (10.4\%) that were not possible to determine (NPD) in at least one of the three examinations were excluded from the calculations of diagnostic accuracy, specificity and sensitivity, rendering 175 ears for inclusion in the study (Figure 1).

\section{Table 3}

Distribution of diagnosis of included ears, $n=175$

\begin{tabular}{lcccc} 
& OM & OTO & V01 & VO2 \\
\hline Normal & $144(82 \%)$ & $140(80 \%)$ & $143(82 \%)$ & $147(84 \%)$ \\
OME & $21(12 \%)$ & $21(12 \%)$ & $23(13 \%)$ & $18(10 \%)$ \\
AOM & $1(1 \%)$ & $4(2 \%)$ & $0(0 \%)$ & $0(0 \%)$ \\
CSOM & $9(5 \%)$ & $10(6 \%)$ & $9(5 \%)$ & $10(6 \%)$
\end{tabular}

$O M=$ otomicroscopy $; O T O=$ otoscopy; $V O 1=$ video-otoscopy $1 ;$ VO2 = video-otoscopy $2 ;$ OME = otitis media with effusion; $A O M=$ acute otitis media; $C S O M=$ chronic suppurative otitis media; 


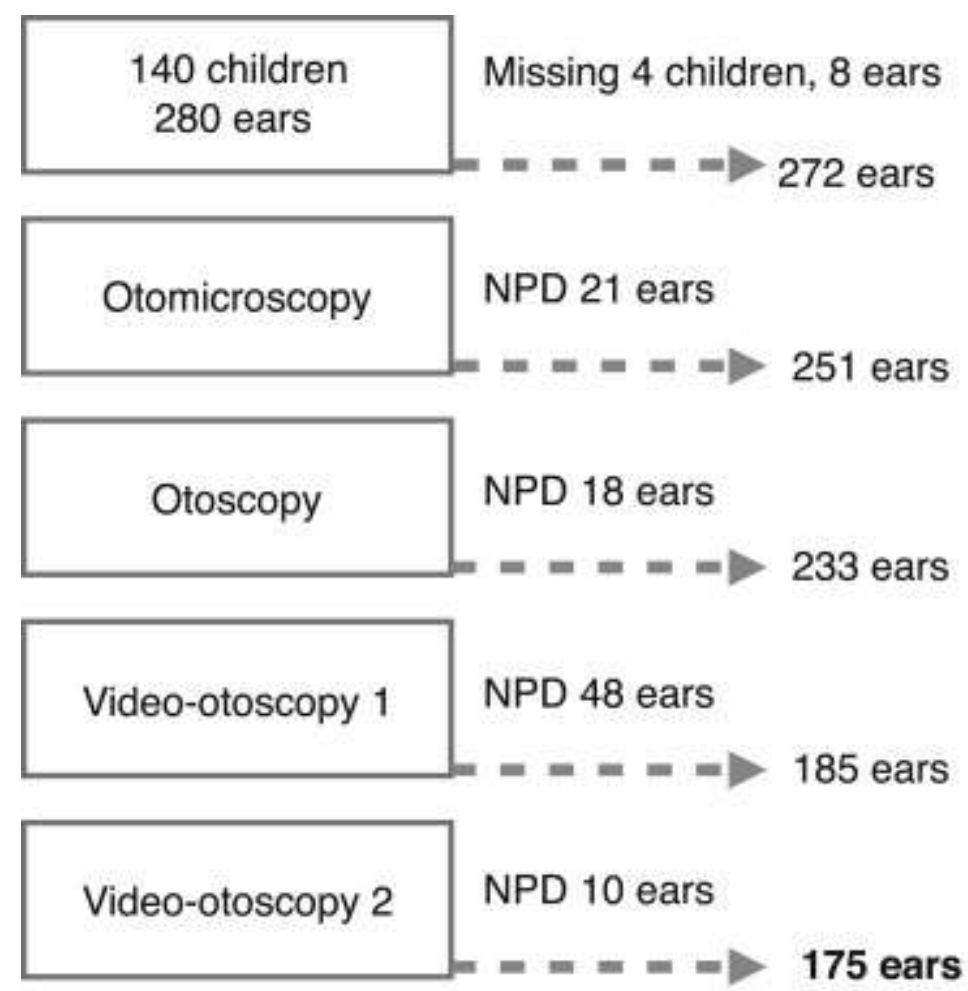

Fig. 1. Flow chart of exclusions for each step.

3.1. Agreement between reference standard and index tests

In $156(89 \%)$ of the otoscopy assessments by the GP, the diagnosis was in concordance with the otologist's assessments compared to $159(91 \%)$ and $165(95 \%)$ of ears at video-otoscopy 1 and 2, respectively. Substantial agreement was found for both video-otoscopy and otoscopy (Table 4).

\section{Table 4}

Distribution of correct diagnoses and diagnostic agreement for otoscopy and videootoscopy compared to otomicroscopy

\begin{tabular}{lcccccc} 
& $\begin{array}{c}\text { Correct } \\
\text { diagnosis }\end{array}$ & $\begin{array}{c}\text { Overall } \\
\text { agreement }\end{array}$ & $\begin{array}{c}\text { Cohen's } \\
\text { kappa }\end{array}$ & $\begin{array}{c}\text { Weighted } \\
\text { kappa }\end{array}$ & SE & 95\% Cl \\
\hline Otoscopy: & $156 / 175$ & $89 \%$ & 0.665 & 0.776 & 0.069 & $0.523-0.807$ \\
& $159 / 175$ & $91 \%$ & 0.704 & 0.769 & 0.069 & $0.566-0.842$ \\
$\begin{array}{l}\text { Video- } \\
\text { otoscopy 1: }\end{array}$ & $165 / 175$ & $94 \%$ & 0.805 & 0.860 & 0.059 & $0.688-0.922$ \\
$\begin{array}{l}\text { Video- } \\
\text { otoscopy 2: }\end{array}$ & & & & & & \\
\hline
\end{tabular}


p=0.533 Otoscopy/Video-otoscopy 1. P=0.039 Otoscopy $/$ Video-otoscopy 2

\subsection{Grading of TM}

The agreement for OMgrade grading's (OMgrading) calculated with weighted kappa was 0.69 between initial otomicroscopy and otoscopy, versus 0.76 and 0.82 , respectively for the two video-otoscopy assessments (Table 5). Findings were not significant except for the higher agreement of video-otoscopic OMgrading at assessment 2 (Table 5, $\mathrm{p}<0.05$ ) as compared with reference standard.

\section{Table 5}

Percentage of correct OMgrade grading compared to otomicroscopy

\begin{tabular}{lcc|ccc} 
& $\begin{array}{c}\text { Correct } \\
\text { grading }\end{array}$ & $\begin{array}{c}\text { Overall percentage of } \\
\text { agreement }\end{array}$ & Weighted kappa & SE & $95 \% \mathrm{Cl}$ \\
\hline Otoscopy: & $148 / 171$ & $87 \%$ & 0.686 & 0.072 & $0.545-0.826$ \\
$\begin{array}{l}\text { Video- } \\
\text { otoscopy 1: }\end{array}$ & $156 / 171$ & $91 \%$ & 0.761 & 0.064 & $0.635-0.887$ \\
$\begin{array}{l}\text { Video- } \\
\text { otoscopy 2: }\end{array}$ & $161 / 171$ & $94 \%$ & 0.819 & 0.048 & $0.726-0.912$ \\
\hline
\end{tabular}

$P=0.088$ Otoscopy-Video-otoscopy 1, $p=0.007$ Otoscopy -Video-otoscopy 2

\subsection{Sensitivity and specificity}

The calculations of sensitivity for otoscopy was 0.81 compared to 0.76 and 0.81 , respectively, for video-otoscopy 1 and 2 (Table 6). We found specificity to be lower, for otoscopy (0.93) compared to that of video-otoscopy 1 and 2 (0.94 and 0.98, respectively). 


\section{Table 6}

Sensitivity and specificity for otoscopy and video-otoscopy diagnosis

\begin{tabular}{lccc} 
& Otoscopy & Video-otoscopy 1 & Video-otoscopy 2 \\
\hline Sensitivity & 0.81 & 0.77 & 0.81 \\
Specificity & 0.93 & 0.94 & 0.98 \\
Positive predictive value & 0.71 & 0.75 & 0.89 \\
Negative predictive value & 0.96 & 0.95 & 0.96 \\
\hline
\end{tabular}

OME, AOM and CSOM grouped together as abnormal. Disease prevalence $17.7 \%$

\section{Discussion}

We found video-otoscopy recordings to have equal or better accuracy compared to traditional otoscopy performed by the same GP with an experienced otologist's diagnosis using otomicroscopy serving as the reference standard. The advantage of video-otoscopy was more apparent in OMgrade assessment of the TM appearance than using conventional OM diagnoses.

We used a test-retest methodology with a four-week interval between video-otoscopy assessment 1 and 2 with images presented in a random order. A four-week interval between face-to-face otoscopy and the video-otoscopy assessment 1 , and another four-week interval for video-otoscopy assessment 2 was applied. Moreover, in this study an experienced otologist made the reference diagnosis using a high quality otomicroscope. No previous study on video-otoscopy has been performed using an otologist's assessments with otomicroscopy as comparison.

Diagnosis of OM is based on symptoms, assessment of the TM appearance together with an evaluation of the middle ear status. A bulging TM or a translucent TM with visible fluid 
levels are sufficient for diagnosis of AOM and OME, respectively. In cases with an opaque $\mathrm{TM}$ in a normal position, assessment with the addition of tympanometry or pneumatic otoscopy would have been desirable. Pneumatic otoscopy or tympanometry was not available at the health clinic at the time of the study. However, previous studies have shown that otomicroscopy alone by an otologist has a sensitivity and specificity of $94 \%$ in detecting middle ear effusion (14).

A limitation of the present study is the high number of normal ears. Our results may therefore not be generalizable to all clinical situations. The shortage of AOM cases in this study combined with a low disease prevalence may have affected the positive predictive value resulting in a lower value. No significant differences were found except for otoscopic grading and video-otoscopy 2. Further studies to compare modern diagnostic methods in OM are required.

The high number of NPD for video-otoscopy was problematic. The kappa values would have been lower for the video-otoscopy evaluations if all NPD's would have been included in this study. Video-otoscopies were performed by a health care assistant with no formal ENT training. To ensure that bias was not introduced due to problems with video captures, the NPD's were excluded from analyses.

The GP's accuracy in video-otoscopic assessments increased between the first and the second assessment. This indicates a learning effect in interpreting the video-otoscopy recordings. More regular use of video-otoscopy may lead to better quality recordings and diagnostic accuracy.

In traditional hand-held otoscopy the examiner gets a restricted and narrow image of the TM and, in addition, a child may sometimes only allow a brief view of the ear canal and TM. 
Video-otoscopy recordings viewed on a large screen by the examiner the patient, however, provide a magnification and good resolution of the TM and can be viewed by the examiner repeatedly in slow motion, or frame-by-frame, which gives a better opportunity to study the TM in detail. This is supported by our results which show a better accuracy for videootoscopy assessments in grading the TM, suggesting that a more detailed examination of the TM was possible. In the study by Blomgren et al (4) the sensitivity for the GP diagnoses of $\mathrm{AOM}$ and $\mathrm{OME}$ was calculated to $82 \%$, whereas the specificity only was $50 \%$ compared to an otologist's assessment. These findings can be compared to $0.81 \%$ and $0.93 \%$, respectively for the GP's otoscopy diagnosis in our study.

Diagnostic accuracy among GP's and pediatricians directly affects over-diagnosis of OM with the possibility of unnecessary use of antibiotics $(4,15-17)$. Whilst too early to conclude that video-otoscopy may improve diagnostic accuracy, our results demonstrate an increase in specificity and positive predictive value with video-otoscopy compared to otoscopy. Videootoscopy appears to be better compared to traditional otoscopy for defining the normal TM, whereas the ability to identify disease does not improve with video-otoscopy. Positive predictive value increased for video-otoscopy in the second assessment, as was the case for agreement in diagnosis and grading, but these findings may have been influenced by the learning effect. Over-diagnosis of AOM is related to low specificity and all methods that can increase specificity are of interest. Tympanometry or pneumatic otoscopy are known to be important to increase specificity $(4,15,16)$. Furthermore, improving diagnostic accuracy depends on clinicians using proper diagnostic criteria with less focus on the redness and more focus on the position of and the transparency of the TM (4). The price range of the videootoscope used in this study is similar or lower than for a conventional wall-mounted otoscope. 
A practical advantage of video-otoscopy is that children may allow examination of the ear more readily if they can see an image on a computer screen. Video-otoscopy also offers the examiner the possibility to show and explain to patients or caregivers about the present ear status. Richards et al (9) used a smartphone otoscope and compared it to a handheld otoscope and found it to be easier to use and suitable for educational purposes. A study comparing patient satisfaction for children examined with conventional otoscopy versus video-otoscopy showed higher patient-centeredness and parental satisfaction with video-otoscopy (18).

In this study video-otoscopy was performed by a health care facilitator with no medical education and with asynchronous assessment by a GP. In rural communities where specialists are scarce, the use of video-otoscopy in a telemedicine setting can give access to professional examinations remotely. Video-otoscopy performed by a health care facilitator has previously been shown to be of adequate quality compared to video-otoscopic examinations performed by a nurse or an otologist $(19,20)$.

\section{Conclusion}

In conclusion, we found that a general practitioners' diagnostic accuracy of video-otoscopy performed by a health care facilitator was good. In our population with low disease prevalence it was similar or better compared to face-to-face hand-held otoscopy performed by the same general practitioner.

\section{Acknowledgements}

The authors wish to thank the patients and the staff at Witkoppen health and welfare centre, Johannesburg, South Africa 


\section{Funding and conflict of interest}

This work was supported by the county of Västerbotten (VLL) and the Swedish Medical Association (SLS). The authors have no conflict of interest

\section{REFERENCES}

1. Acuin J. Chronic suppurative otitis media. Clin Evid. 2004(12):710-29.

2. Monasta L, Ronfani L, Marchetti F, Montico M, Vecchi Brumatti L, Bavcar A, et al. Burden of disease caused by otitis media: systematic review and global estimates. PLoS One. 2012;7(4):e36226.

3. Shekelle P, Takata G, Chan LS, Mangione-Smith R, Corley PM, Morphew T, et al. Diagnosis, natural history, and late effects of otitis media with effusion. Evid Rep Technol Assess (Summ). 2002(55):1-5.

4. Blomgren K, Pitkaranta A. Is it possible to diagnose acute otitis media accurately in primary health care? Fam Pract. 2003;20(5):524-7.

5. Lieberthal AS, Carroll AE, Chonmaitree T, Ganiats TG, Hoberman A, Jackson MA, et al. The diagnosis and management of acute otitis media. Pediatrics. 2013;131(3):e96499.

6. $\quad$ Fagan JJ, Jacobs M. Survey of ENT services in Africa: need for a comprehensive intervention. Glob Health Action. 2009;2.

$7 . \quad$ Biagio L, Swanepoel de W, Laurent C, Lundberg T. Video-otoscopy recordings for diagnosis of childhood ear disease using telehealth at primary health care level. Journal of telemedicine and telecare. 2014;20(6):300-6.

8. Lundberg T, Westman G, Hellstrom S, Sandstrom H. Digital imaging and telemedicine as a tool for studying inflammatory conditions in the middle ear--evaluation of image quality and agreement between examiners. International journal of pediatric otorhinolaryngology. 2008;72(1):73-9.

9. Richards JR, Gaylor KA, Pilgrim AJ. Comparison of traditional otoscope to iPhone otoscope in the pediatric ED. The American journal of emergency medicine. 2015;33(8):1089-92.

10. Lundberg T, Biagio L, Laurent C, Sandstrom H, Swanepoel de W. Remote evaluation of video-otoscopy recordings in an unselected pediatric population with an otitis media scale. International journal of pediatric otorhinolaryngology. 2014;78(9):1489-95. 11. Biagio L, Swanepoel D, Laurent C, Lundberg T. Paediatric otitis media at a primary healthcare clinic in South Africa. S Afr Med J. 2014;104(6):431-5.

12. Gates GA, Klein JO, Lim DJ, Mogi G, Ogra PL, Pararella MM, et al. Recent advances in otitis media. 1. Definitions, terminology, and classification of otitis media. Ann Otol Rhinol Laryngol Suppl. 2002;188:8-18.

13. Lundberg T, Hellstrom S, Sandstrom H. Development and validation of a new grading scale for otitis media. Pediatr Infect Dis J. 2013;32(4):341-5.

14. Young DE, Ten Cate WJ, Ahmad Z, Morton RP. The accuracy of otomicroscopy for the diagnosis of paediatric middle ear effusions. International journal of pediatric otorhinolaryngology. 2009;73(6):825-8.

15. Asher E, Leibovitz E, Press J, Greenberg D, Bilenko N, Reuveni H. Accuracy of acute otitis media diagnosis in community and hospital settings. Acta Paediatr.

2005;94(4):423-8. 
16. Blomgren K, Pohjavuori S, Poussa T, Hatakka K, Korpela R, Pitkaranta A. Effect of accurate diagnostic criteria on incidence of acute otitis media in otitis-prone children. Scand J Infect Dis. 2004;36(1):6-9.

17. Pichichero ME, Poole MD. Comparison of performance by otolaryngologists, pediatricians, and general practioners on an otoendoscopic diagnostic video examination. International journal of pediatric otorhinolaryngology. 2005;69(3):361-6.

18. Rimon O, Avraham Y, Sharabi-Nov A, Luder A, Krupik D, Gilbey P. Videootoscopy in children and patient-centered care: A randomized, controlled study. International journal of pediatric otorhinolaryngology. 2015;79(12):2286-9.

19. Biagio L, Swanepoel de W, Adeyemo A, Hall JW, 3rd, Vinck B. Asynchronous video-otoscopy with a telehealth facilitator. Telemed J E Health. 2013;19(4):252-8.

20. Smith AC, Perry C, Agnew J, Wootton R. Accuracy of pre-recorded video images for the assessment of rural indigenous children with ear, nose and throat conditions. Journal of telemedicine and telecare. 2006;12(suppl 3):76-80. 